\title{
Catalytic Enantioselective Approach to the Stereodivergent Synthesis of (+)-Lasubines I and II
}

Olga García Mancheño, Ramón Gómez Arrayás, Javier Adrio, and Juan C. Carretero* Departamento de Química Orgánica, Universidad Autónoma de Madrid. Cantoblanco 28049 Madrid, Spain.

juancarlos.carretero@uam.es

\section{Supporting Information}

General: Aldehydes, arylsulfonamides, copper salts and Danishefsky's diene were purchased from commercial suppliers and were used without further purification. The preparation of metal complex 3 has been previously reported by us. ${ }^{1}$ Racemic piperidone 1 was obtained using $\mathrm{AgClO}_{4}(20 \mathrm{~mol} \%)$ as catalyst. Toluene and $\mathrm{CH}_{2} \mathrm{Cl}_{2}$ were dried over microwave activated $4 \AA$ MS. Reactions were monitored by thin-layer chromatography using $0.25 \mathrm{~mm}$ silica gel plates $(60$ 230-400 mesh). 230-400 Mesh silica gel was used for flash column chromatography. NMR spectra were recorded on Brucker AC-200 or AC-300 instruments in $\mathrm{CDCl}_{3}$ (calibrated at 7.26 and $77.0 \mathrm{ppm}$ for ${ }^{1} \mathrm{H}$ and ${ }^{13} \mathrm{C}$ experiments, respectively). Mass spectra were determined on a Hewlett-Packard HP-5985 mass spectrometer at $70 \mathrm{eV}$ ionising voltage (EI) or under fast atom bombardment (FAB) conditions. IR spectra were taken on a Bruker Vector-22 and were recorder as $\mathrm{KBr}$ pellets or in solution). Melting points were determined in open-end capillary tubes on a GallemKamp apparatus. Optical rotations were measured on a Perkin-Elmer 241C polarimeter. High-Performance Liquid Chromatography (HPLC) was conducted on an Agilent 1100 instrument, using Daicel Chiralcel OD columns.

\section{$(4 R, 10 R)-4-(3,4-D i m e t h o x y p h e n y l) q u i n o l i z i d i n-2-o n e(7)$}<smiles>COc1ccc([C@@H]2CC(=O)C[C@H]3CCCCN23)cc1OC</smiles>

To a solution of $6(30 \mathrm{mg}, 0.070 \mathrm{mmol})$ in AcOEt $(2 \mathrm{~mL})$ a $1 \mathrm{M}$ solution of $\mathrm{SnCl}_{4}$ in $\mathrm{CH}_{2} \mathrm{Cl}_{2}(0.352 \mathrm{mmol})$ was added, the mixture was stirred for $3 \mathrm{~h}$ at room temperature and the solvent was evaporated at reduced pressure. The residue was dissolved in THF (3 mL), a saturated aqueous solution of $\mathrm{K}_{2} \mathrm{CO}_{3}(5 \mathrm{~mL})$ was added, and the reaction was stirred $12 \mathrm{~h}$ at room temperature. The combined organic layers were washed with brine, dried over $\mathrm{MgSO}_{4}$ and filtered. After evaporation of the solvent, the residue was purified by flash chromatography $\left(\mathrm{CH}_{2} \mathrm{Cl}_{2} / 15 \%\right.$ $\mathrm{MeOH}$ ), to afford 7 (14 mg, 70\%, colorless oil). 
$[\alpha]_{\mathrm{D}}{ }^{20}=+71\left(\mathrm{c} 0.25, \mathrm{CHCl}_{3}\right) ;$ Lit. $^{2}(4 S, 10 S)-7[\alpha]_{\mathrm{D}}{ }^{20}=-86\left(c \quad 0.33, \mathrm{CHCl}_{3}\right)$.

${ }^{1} \mathrm{H}-\mathrm{NMR}(300 \mathrm{MHz}): \delta$ 7.00-6.75 (m, 3H, Ar), 3.90 (s, 3H, MeO), 3.87 (s, 3H, MeO), 3.20 (dd, $1 \mathrm{H}, J=12.1,2.8 \mathrm{~Hz}, \mathrm{H}-4), 2.85-2.19(\mathrm{~m}, 6 \mathrm{H}), 1.80-1.15(\mathrm{~m}, 7 \mathrm{H}) .{ }^{13} \mathrm{C}-\mathrm{NMR}(75 \mathrm{MHz}): \delta 207.9$, $149.4,148.4,135.2,119.5,111.1,109.9,70.0,62.5,56.0,55.9,52.8,50.9,48.8,34.4,25.9$, 24.2. IR $\left(\mathrm{CHCl}_{3}\right): v 2933,1715,1517,1261,1027$.

$(2 R, 4 R, 10 R)-4-(3,4-D i m e t h o x y p h e n y l) q u i n o l i z i d i n-2-o l:(+)-L a s u b i n e ~ I{ }^{2,3,4}$<smiles>COc1ccc([C@@H]2C[C@H](O)C[C@H]3CCCCN23)cc1OC</smiles>

To a solution of $7(5.0 \mathrm{mg}, 0.017 \mathrm{mmol})$ in THF $(0.6 \mathrm{~mL})$ at -78 ${ }^{\circ} \mathrm{C}$ a $1 \mathrm{M}$ solution of L-selectride ${ }^{\circledR}$ in THF $(20 \mu \mathrm{L}, 0.020 \mathrm{mmol})$ was added. The mixture was stirred at $-78^{\circ} \mathrm{C}$ for $3 \mathrm{~h}$ and $\mathrm{MeOH}$ $(20 \mu \mathrm{l})$ was added. The resulting suspension was filtered over Cetite and was treated with a $2 \mathrm{~N}$ aqueous solution of $\mathrm{NaOH}$ $(1.5 \mathrm{~mL})$. The organic phase was separated and the aqueous phase was extracted with $\mathrm{CH}_{2} \mathrm{Cl}_{2}(10 \mathrm{~mL})$. The combined organic layers were washed with brine, dried over $\mathrm{MgSO}_{4}$ and filtered. After evaporation of the solvent, the residue was purified by flash chromatography $\left(\mathrm{CH}_{2} \mathrm{Cl}_{2} / 20 \% \mathrm{MeOH}\right)$ to afford Lasubine II (3.2 $\mathrm{mg}, 65 \%$, colorless oil). $[\alpha]_{D}{ }^{20}=+50$ (c 0.3, MeOH); Lit $^{2}(-)$-Lasubine II $[\alpha]_{D}{ }^{25}=-53$ (c $\left.0.13, \mathrm{MeOH}\right) ;$ Lit. $^{4}[\alpha]_{\mathrm{D}}{ }^{25}=-50$ (c $0.37, \mathrm{MeOH})$.

${ }^{1} \mathrm{H}-\mathrm{NMR}(300 \mathrm{MHz}): \delta$ 6.98-6.70 (m, 3H, Ar), 4.19-4.10 (m, $\left.1 \mathrm{H}, \mathrm{H}-2\right), 3.90\left(\mathrm{~s}, 3 \mathrm{H}, \mathrm{CH}_{3} \mathrm{O}\right), 3.87$ (s, 3H, $\mathrm{CH}_{3} \mathrm{O}$ ), 3.31 (dd, $\left.1 \mathrm{H}, J=8.2,3.7 \mathrm{~Hz}, \mathrm{H}-4\right), 2.69(\mathrm{~d}, 1 \mathrm{H}, \mathrm{J}=11.2 \mathrm{~Hz}), 2.48-2.27(\mathrm{~m}, 1 \mathrm{H})$, 1.95-1.20 (m, 12H). ${ }^{13} \mathrm{C}-\mathrm{NMR}(75 \mathrm{MHz}): \delta$ 149.0, 147.8, 137.3, 119.7, 111.0, 110.5, 65.1, 63.4, 56.4, 56.0, 55.9, 53.2, 42.8, 40.4, 33.7, 26.2, 24.9. IR $\left(\mathrm{CH}_{2} \mathrm{Cl}_{2}\right): v 3560,2927,1516,1261$.

\section{(4R,10S)-4-(3,4-Dimethoxyphenyl)quinolizidin-2-one (10)}

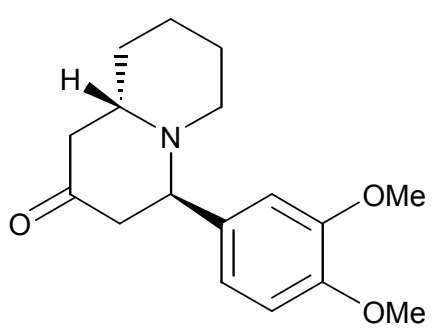

To a solution of $9(50.0 \mathrm{mg}, 0.12 \mathrm{mmol})$ in degassed benzene ( 3 $\mathrm{mL})$ at reflux a solution of $\mathrm{Bu}_{3} \mathrm{SnH}(65 \mu \mathrm{L}, 0.24 \mathrm{mmol})$ and AIBN $(2.0 \mathrm{mg}, 0.01 \mathrm{mmol})$ in benzene $(3 \mathrm{~mL})$ was slowly added. The mixture was refluxed for $8 \mathrm{~h}$, cooled to room temperature and concentrated. The residue was purified by flash chromatography (hexane to $\mathrm{AcOEt} / \mathrm{Et}_{2} \mathrm{O}$ 1:1) to afford 10 (24 mg, 69\%, yellow oil) and 11 (5.6 mg, 16\%, white oil).

$[\alpha]_{D}^{25}=-12\left(c 0.32, \mathrm{CHCl}_{3}\right) ;$ Lit. $^{5}[\alpha]_{D}{ }^{25}=+10.8\left(c 1.31, \mathrm{CHCl}_{3}\right) ; \mathrm{Lit}^{3}[\alpha]_{D}{ }^{25}=+9.5\left(c 3.1, \mathrm{CHCl}_{3}\right)$.

${ }^{1} \mathrm{H}-\mathrm{NMR}(300 \mathrm{MHz}): \delta 6.83-6.77(\mathrm{~m}, 1 \mathrm{H}, \mathrm{Ar}), 6.70-6.63(\mathrm{~m}, 2 \mathrm{H}, \mathrm{Ar}), 4.23(\mathrm{dd}, 1 \mathrm{H}, \mathrm{J}=6.3,4.0 \mathrm{~Hz}$, $\mathrm{H}-4), 3.88\left(\mathrm{~s}, 3 \mathrm{H}, \mathrm{CH}_{3} \mathrm{O}\right), 3.85\left(\mathrm{~s}, 3 \mathrm{H}, \mathrm{CH}_{3} \mathrm{O}\right), 2.95-2.80\left(\mathrm{~m}, 3 \mathrm{H}, \mathrm{CH}_{2}+1 \mathrm{H}\right), 2.67-2.50(\mathrm{~m}, 2 \mathrm{H}$, $\mathrm{CH}_{2}$ ), 2.36 (ddd, $1 \mathrm{H}, \mathrm{J}=15.0,8.9,1.4 \mathrm{~Hz}, \mathrm{CH}_{2}$ ), 2.19 (td, $1 \mathrm{H}, \mathrm{J}=11.7,3.4 \mathrm{~Hz}, \mathrm{CH}_{2}$ ), 1.80-1.32 $(\mathrm{m}, 5 \mathrm{H}), 1.26-1.14(\mathrm{~m}, 1 \mathrm{H}) .{ }^{13} \mathrm{C}-\mathrm{NMR}(50 \mathrm{MHz}): \delta 209.6,148.7,148.4,131.6,120.9,111.8$, 110.7, 63.9, 55.9, 55.8, 54.3, 51.3, 47.6, 46.8, 31.9, 24.1, 23.4. IR $\left(\mathrm{CH}_{2} \mathrm{Cl}_{2}\right): v 2940,1715$, 1520, 1259. MS (El+): $289\left(M^{+}, 48\right), 206$ (33), 160 (100), 84 (54). 


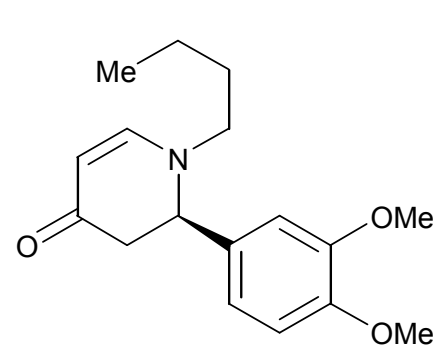

$[\alpha]_{D}{ }^{25}=-92\left(c 0.3, \mathrm{CHCl}_{3}\right)$.

${ }^{1} \mathrm{H}-\mathrm{NMR}(300 \mathrm{MHz}): \delta 7.12(\mathrm{~d}, 1 \mathrm{H}, J=7.5 \mathrm{~Hz}, \mathrm{H}-6), 6.82(\mathrm{~s}, 3 \mathrm{H}$, Ar), $5.01(\mathrm{~d}, 1 \mathrm{H}, J=7.5 \mathrm{~Hz}, \mathrm{H}-5), 4.53(\mathrm{dd}, 1 \mathrm{H}, J=8.5,6.9, \mathrm{~Hz}, \mathrm{H}-$ 2), $3.88\left(\mathrm{~s}, 3 \mathrm{H}, \mathrm{CH}_{3} \mathrm{O}\right), 3.86\left(\mathrm{~s}, 3 \mathrm{H}, \mathrm{CH}_{3} \mathrm{O}\right), 3.10-3.02(\mathrm{~m}, 2 \mathrm{H}$, $\left.\mathrm{CH}_{2}\right), 2.82\left(\mathrm{dd}, 1 \mathrm{H}, \mathrm{J}=16.4,6.9 \mathrm{~Hz}, \mathrm{CH}_{2}\right), 2.67(\mathrm{dd}, 1 \mathrm{H}, \mathrm{J}=16.4$, $\left.8.7 \mathrm{~Hz}, \mathrm{CH}_{2}\right), 1.50\left(\mathrm{q}, 2 \mathrm{H}, \mathrm{J}=7.4 \mathrm{~Hz}, \mathrm{CH}_{2}\right), 1.37-1.16(\mathrm{~m}, 2 \mathrm{H}$, $\left.\mathrm{CH}_{2}\right), 0.89$ (t, 3H, J=7.3 Hz, CH$)_{3}{ }^{13} \mathrm{C}-\mathrm{NMR}(50 \mathrm{MHz}): \delta 109.4,154.0,149.5,149.0,131.4$, 119.5, 111.3, 109.9, 98.0, 61.0, 56.0, 53.1, 44.0, 30.8, 19.8, 13.7. IR $\left(\mathrm{CH}_{2} \mathrm{Cl}_{2}\right): v 2958,1636$, 1583, 1516, 1261. MS (APCI+, 90V): $290\left(\mathrm{M}^{+}\right)$.

$(2 R, 4 R, 10 S)-4-(3,4-D i m e t h o x y p h e n y l) q u i n o l i z i d i n-2-o l:(+)-L a s u b i n e ~ I^{3,6}$<smiles>COc1ccc([C@@H]2C[C@H](O)C[C@H]3CCCCN23)cc1OC</smiles>

To a solution of $10(20.0 \mathrm{mg}, 0.07 \mathrm{mmol})$ in THF $(2 \mathrm{~mL})$ at -78 ${ }^{\circ} \mathrm{C}$ a $1 \mathrm{M}$ solution of L-Selectride ${ }^{\circledR}$ in THF $(83 \mu \mathrm{L}, 0.08 \mathrm{mmol})$ was added. The mixture was stirred at $-78^{\circ} \mathrm{C}$ for $3 \mathrm{~h}$ and $\mathrm{MeOH}$ $(80 \mu \mathrm{l})$ was added. The resulting suspension was filtered over Cetite and was treated with a $2 \mathrm{~N}$ aqueous solution of $\mathrm{NaOH}$ $(1.5 \mathrm{~mL})$. The organic layer was separated and the aqueous phase was extracted with $\mathrm{CH}_{2} \mathrm{Cl}_{2}(10 \mathrm{~mL})$. The combined organic phase was washed with brine, dried over $\mathrm{MgSO}_{4}$ and filtered. After evaporation of the solvent, the residue was purified by flash chromatography (acetone) to afford Lasubine I (16.1 mg, 79\%).

$[\alpha]_{\mathrm{D}}{ }^{25}=+6.2$ (c 0.34, MeOH); Lit. $^{3}(-)$-Lasubine $[\alpha]_{\mathrm{D}}{ }^{25}=-6.5$ (c 2.60, MeOH); Lit. $^{6 \mathrm{~b}}[\alpha]_{\mathrm{D}}{ }^{25}=-7.0$ (c $0.37, \mathrm{MeOH})$.

${ }^{1} \mathrm{H}-\mathrm{NMR}(300 \mathrm{MHz}): \delta 6.92-6.76(\mathrm{~m}, 3 \mathrm{H}, \mathrm{Ar}$ ), 4.18 (sept., $1 \mathrm{H}, J=4.5 \mathrm{~Hz}, \mathrm{H}-2), 4.09(\mathrm{t}, 1 \mathrm{H}, J=4.9$ $\mathrm{Hz}, \mathrm{H}-4), 3.87$ (s, 3H, $\mathrm{CH}_{3} \mathrm{O}$ ), $3.86\left(\mathrm{~s}, 3 \mathrm{H}, \mathrm{CH}_{3} \mathrm{O}\right), 3.03-2.88(\mathrm{~m}, 1 \mathrm{H}), 2.71(\mathrm{dt}, 1 \mathrm{H}, \mathrm{J}=11.7,3.4$ $\mathrm{Hz}), 2.25(\mathrm{td}, 1 \mathrm{H}, \mathrm{J}=11.1,3.2 \mathrm{~Hz}), 2.14-1.88(\mathrm{~m}, 3 \mathrm{H}), 1.77-1.38(\mathrm{~m}, 7 \mathrm{H}), 1.34-1.18(\mathrm{~m}, 1 \mathrm{H}) .{ }^{13} \mathrm{C}-$ NMR (50 MHz): $\delta$ 148.8, 147.9, 135.4, 120.5, 111.9, 110.8, 65.1, 61.9, 55.9, 55.8, 53.8, 51.1, 40.2, 40.0, 32.4, 24.6, 24.0. IR $\left(\mathrm{CH}_{2} \mathrm{Cl}_{2}\right): v 3383,2932,1515,1260,1028 . \mathbf{M S}$ (EI+): $291\left(\mathrm{M}^{+}\right.$, 88), 164 (100), 154 (91), 110 (47), 84 (42). 


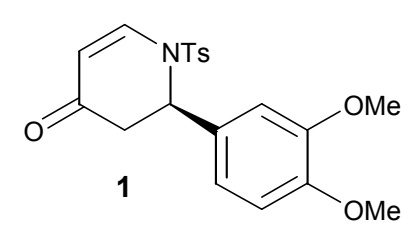

\section{Spectra collection}
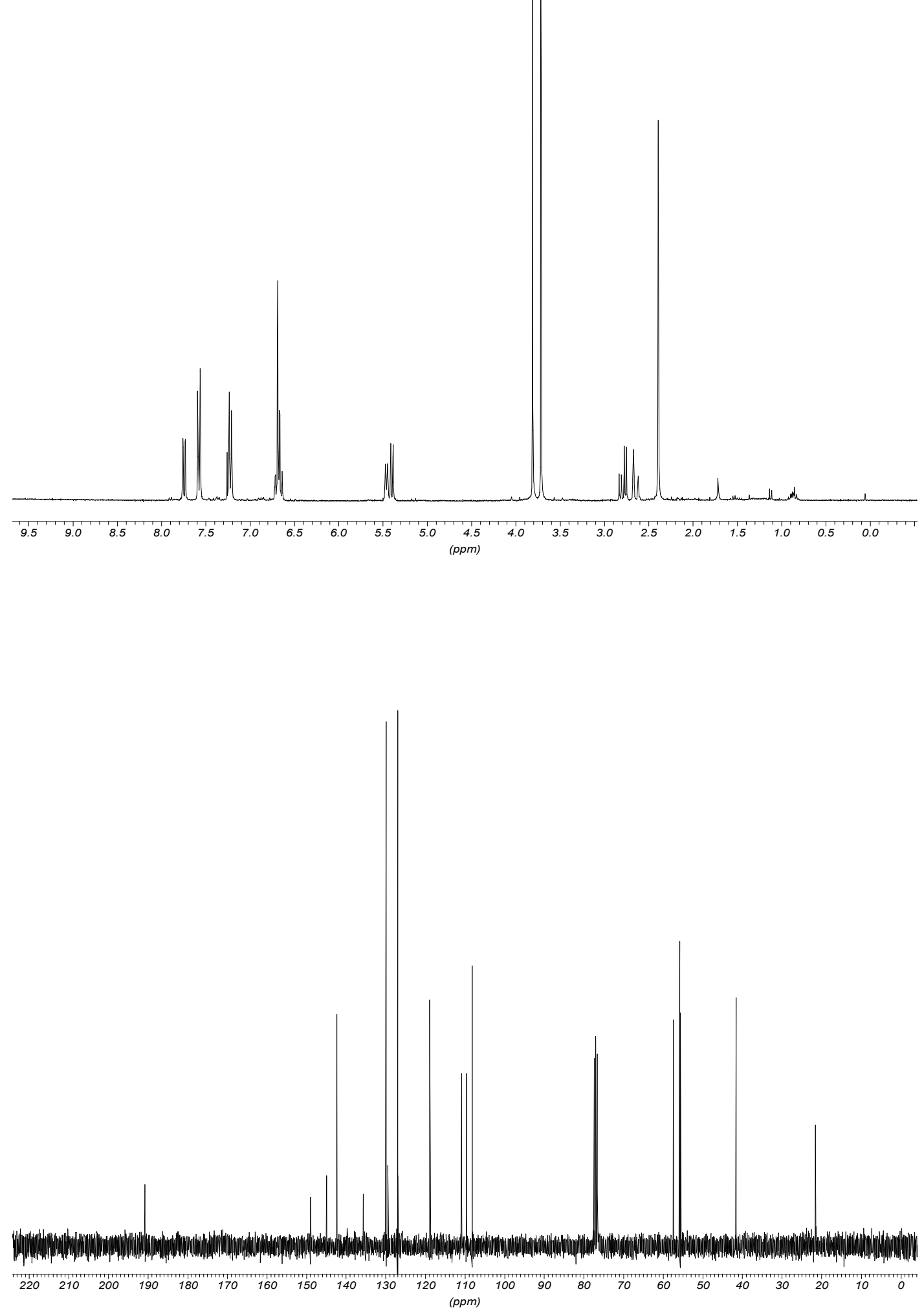

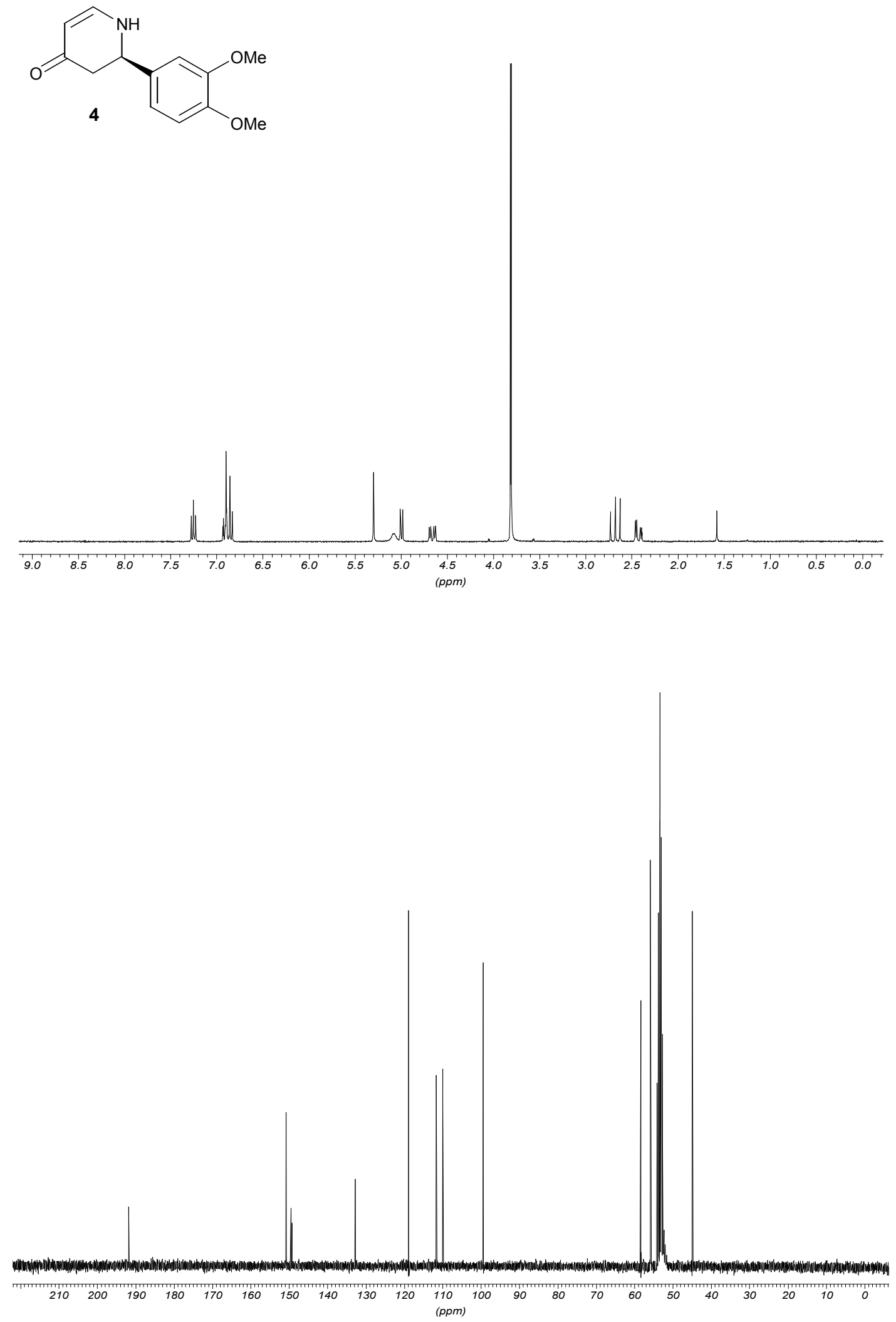
<smiles>COc1ccc(C2CC(=O)C=CN2C(=O)c2ccccc2)cc1OC</smiles>

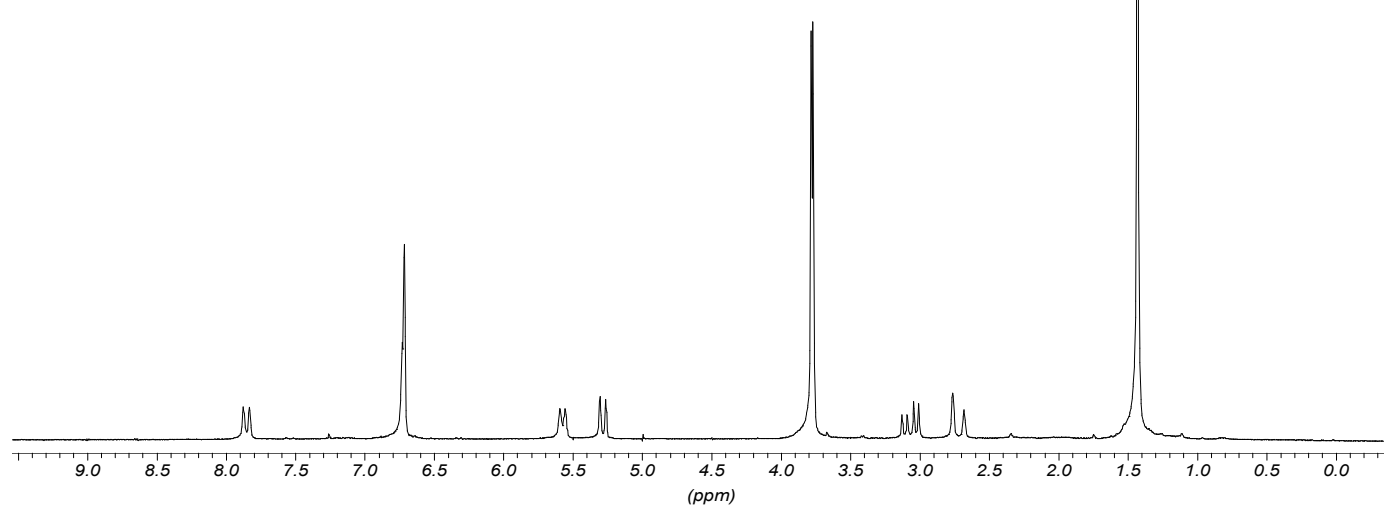

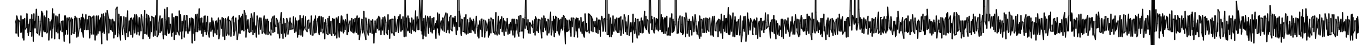

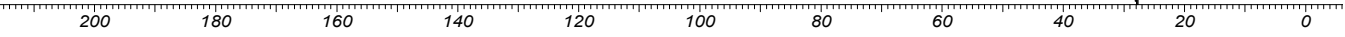

(ppm) 

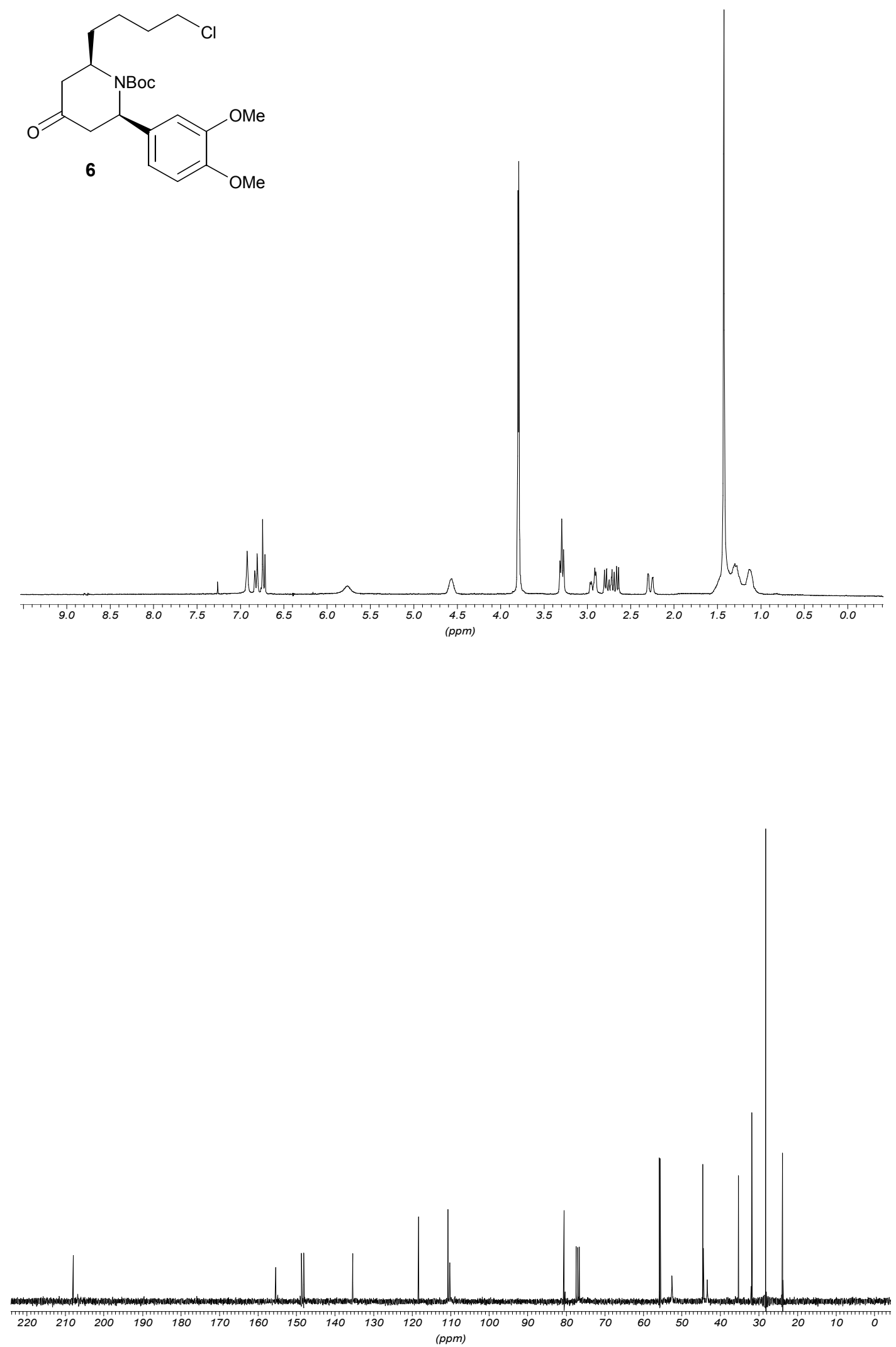
<smiles>COc1ccc([C@@H]2CC(=O)C[C@@H]3CCCCN32)cc1OC</smiles>
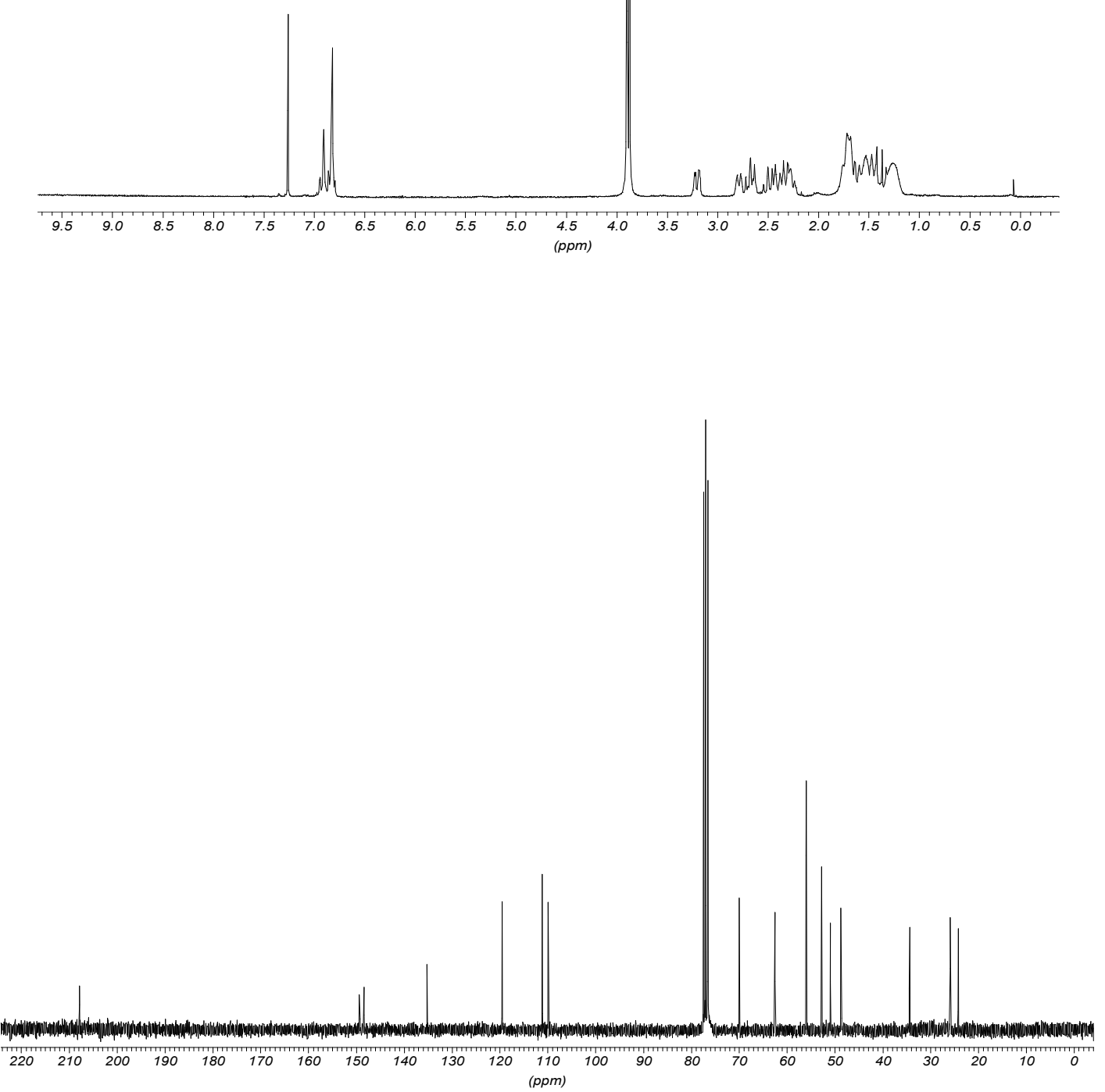
<smiles>COc1ccc([C@@H]2C[C@H](O)C[C@@H]3CCCCN32)cc1OC</smiles>

(+)-Lasubine II

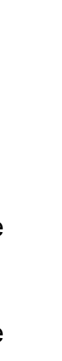
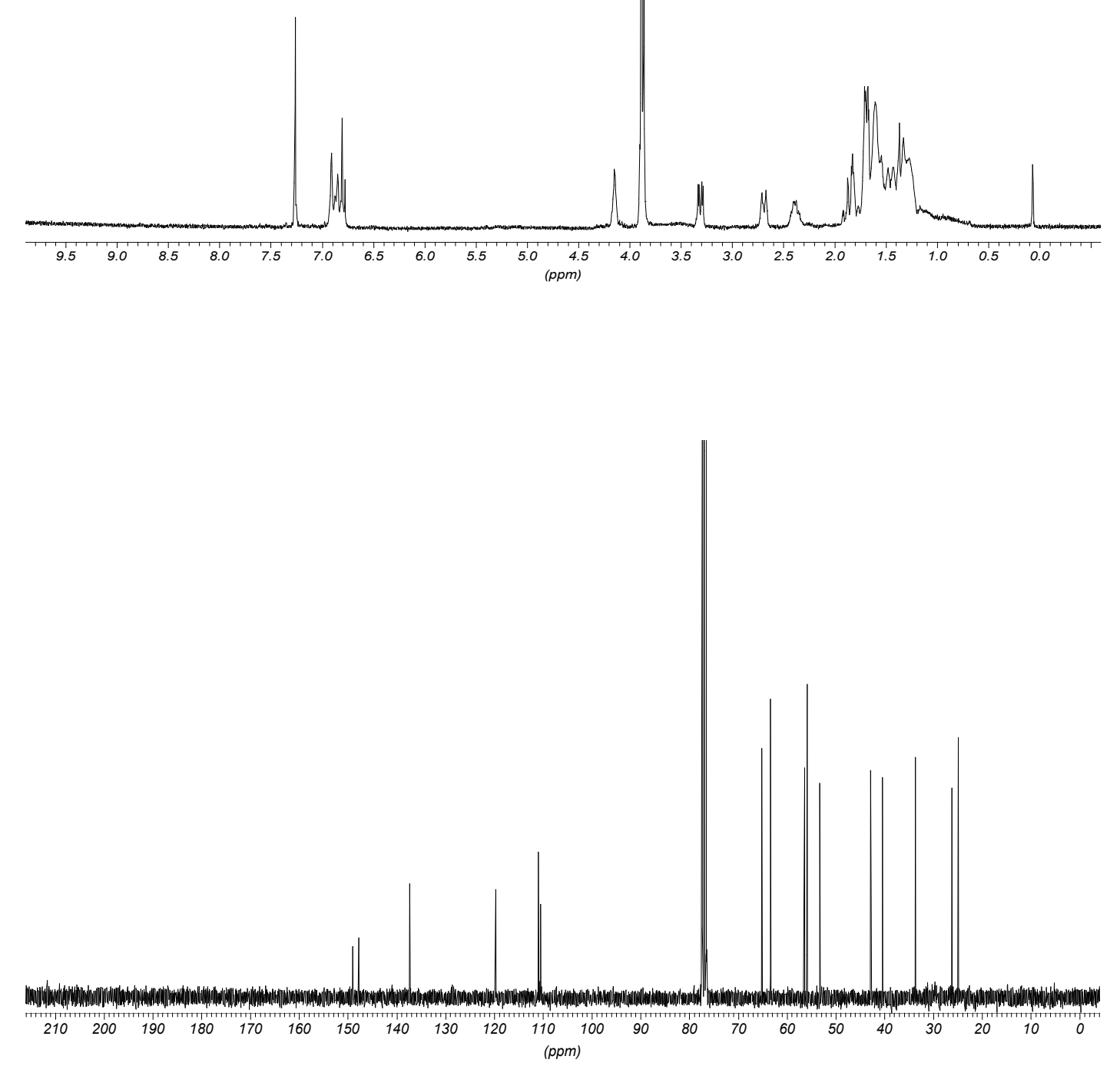
<smiles>COc1ccc([C@@H]2CC(=O)C=CN2CCCCCl)cc1OC</smiles>
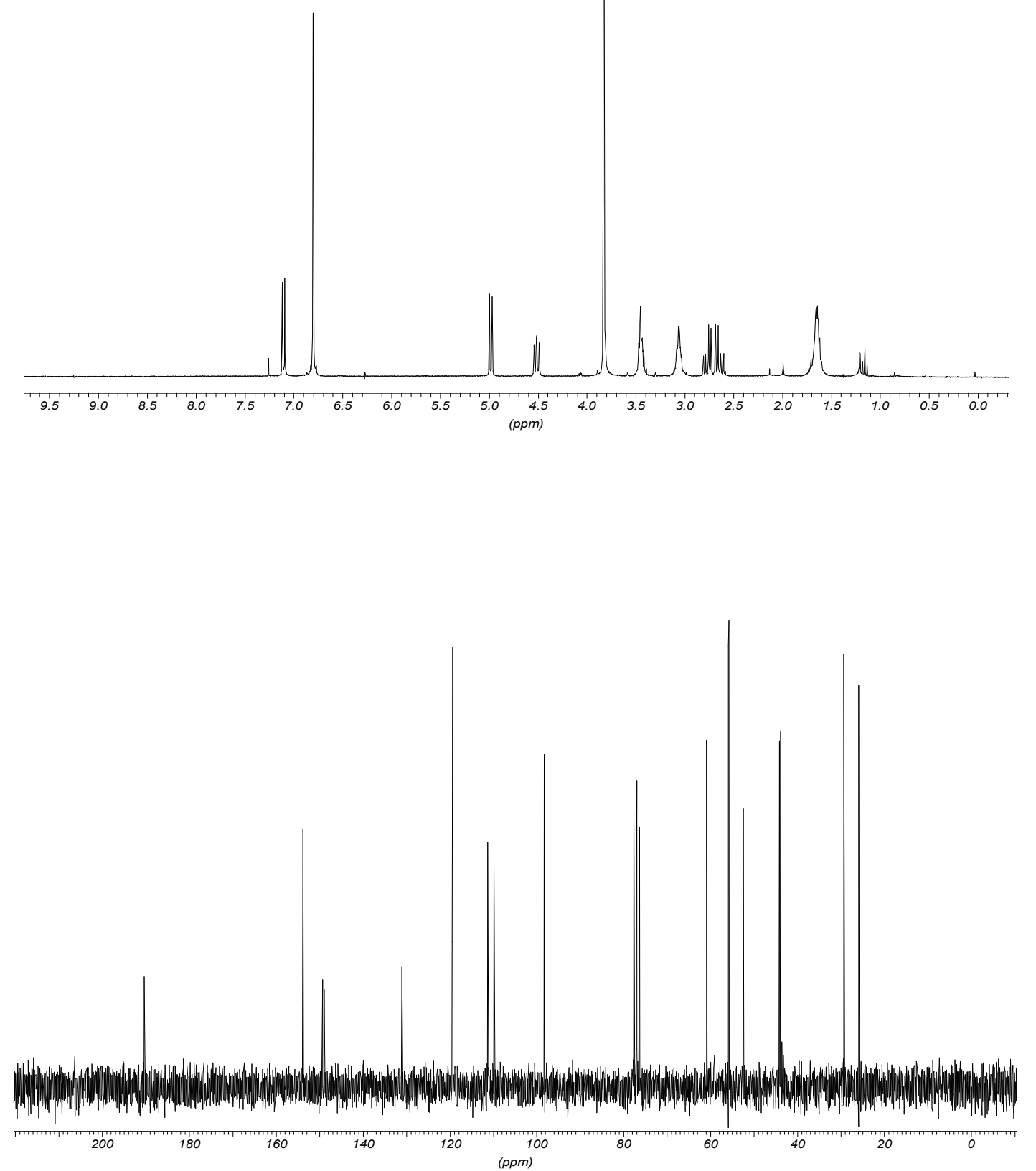

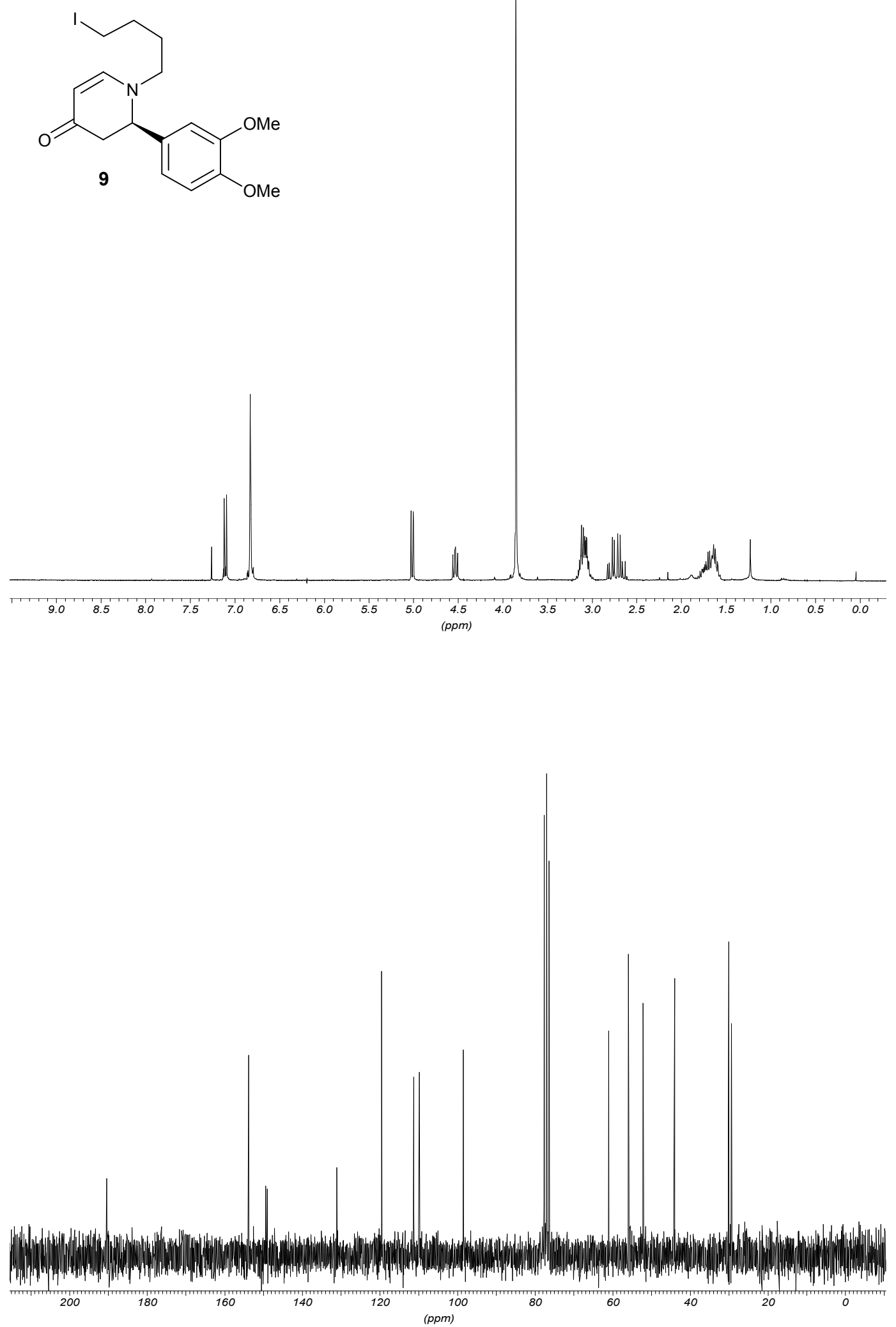
<smiles>COc1ccc([C@@H]2CC(=O)C[C@@H]3CCCCN32)cc1OC</smiles>
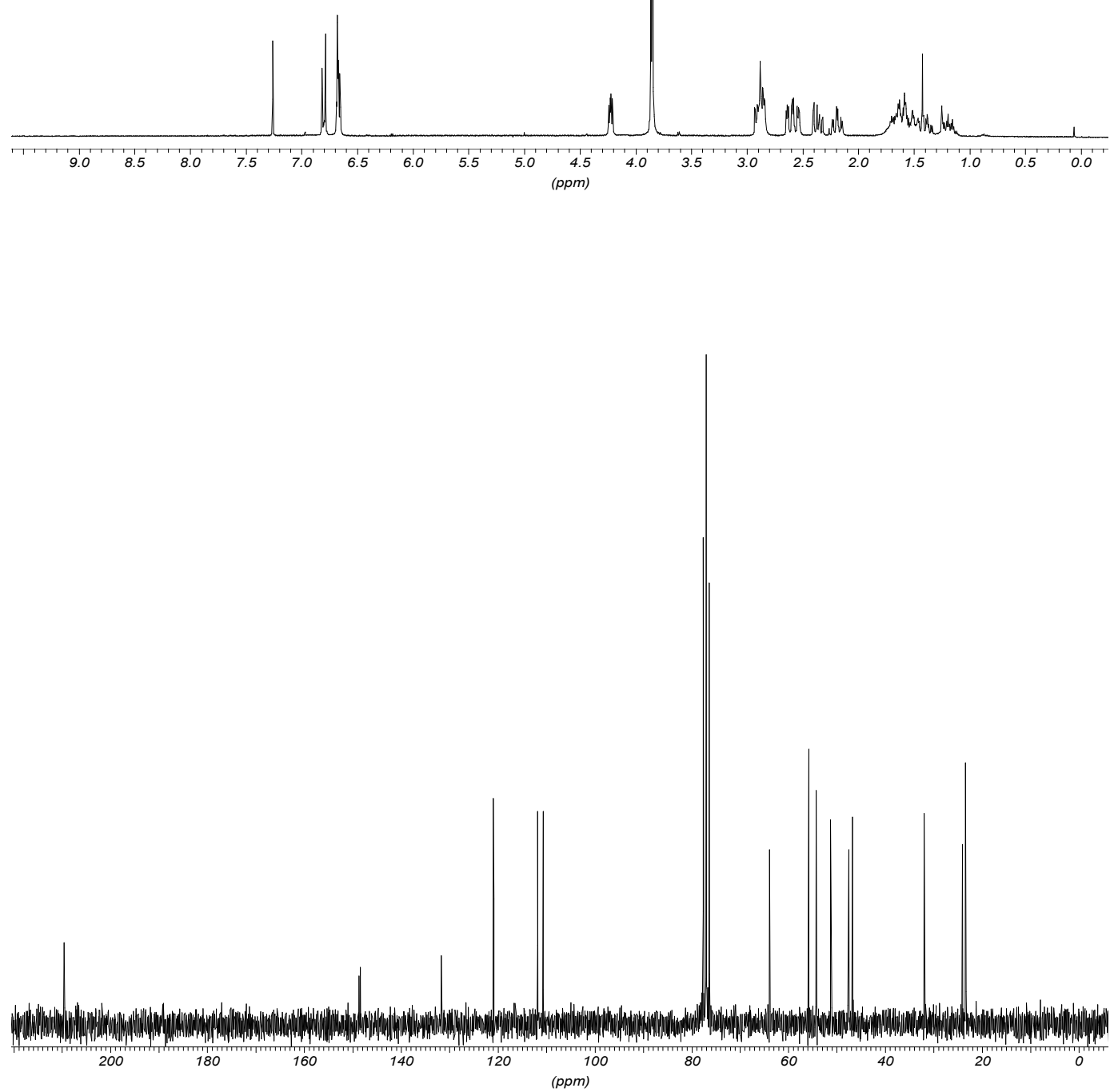
<smiles>CCCCN1C=CC(=O)C[C@H]1c1ccc(OC)c(OC)c1</smiles>
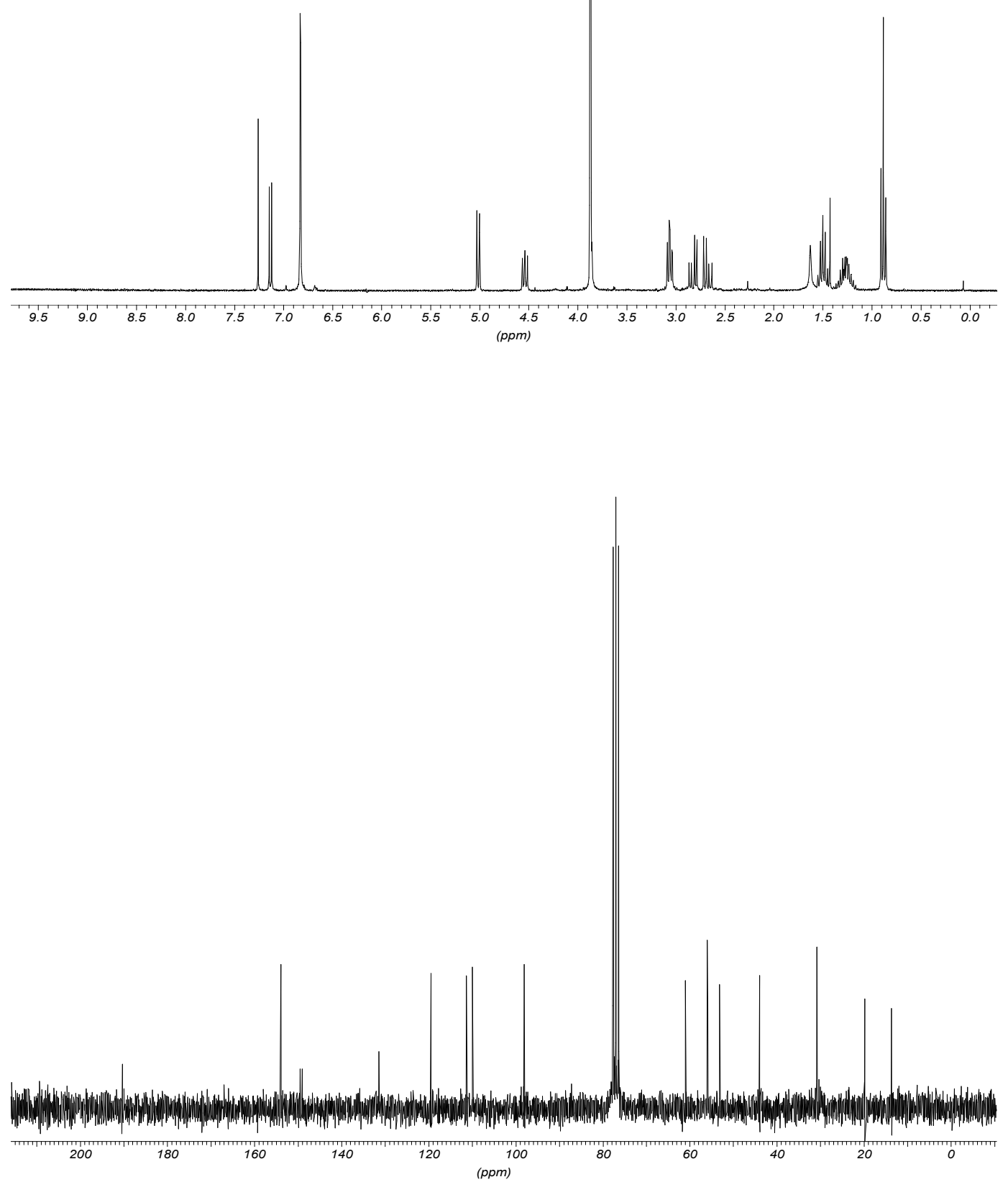
<smiles>COc1ccc(C2C[C@H](O)C[C@H]3CCCCN23)cc1OC</smiles>

(+)-Lasubine I
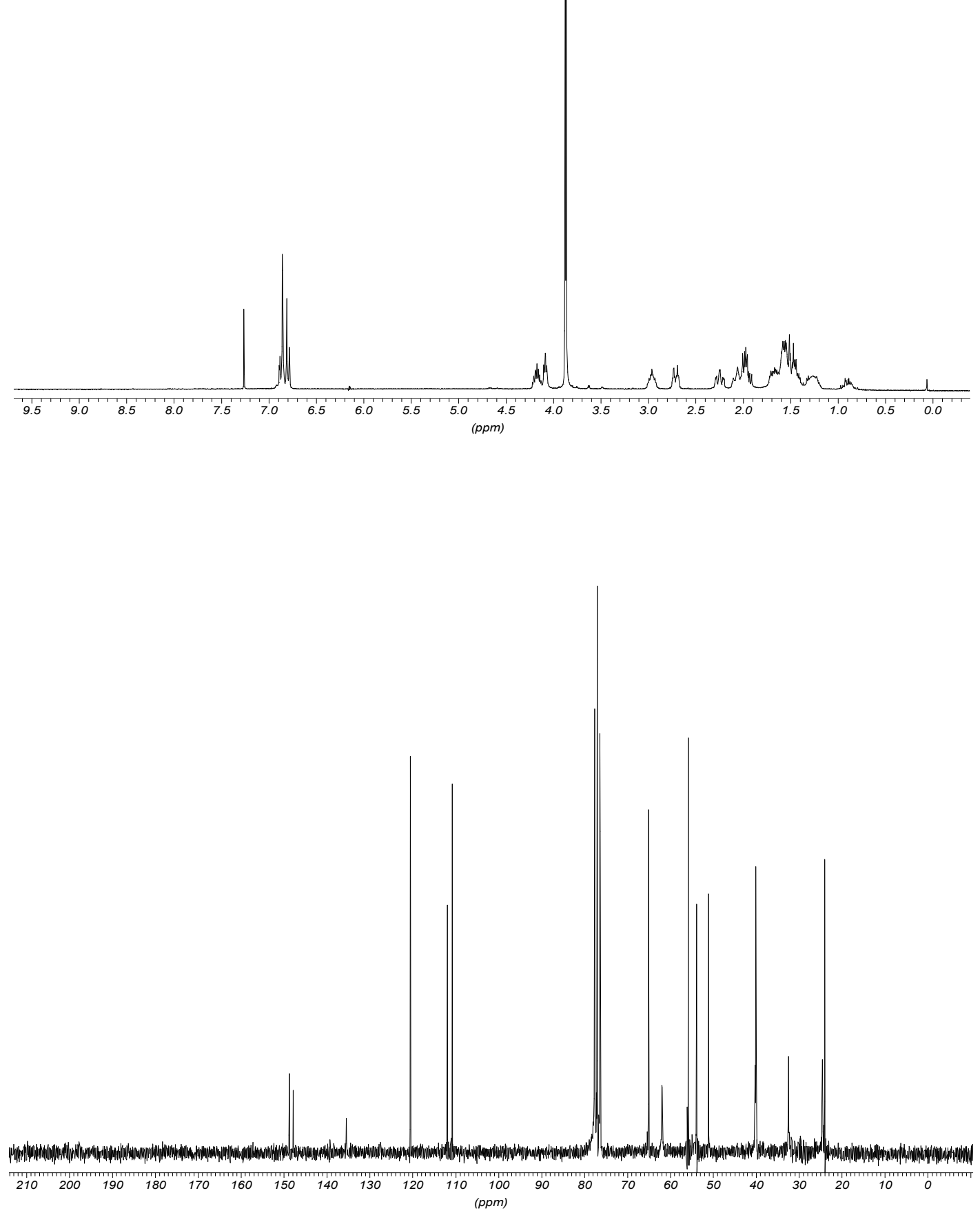


\section{References}

1 García-Mancheño, O.; Gómez-Arrayás, R. Carretero, J. C. J. Am. Chem. Soc. 2004, 126, 456-457. 2 Back, T. G.; Hamilton, M. D. Org. Lett. 2002, 4, 1779.

3 Chalard, P.; Remuson, R.; Gelas-Mialhe, Y.; Gramain, J-C. Tetrahedron: Asymmetry 1998, 9, 4361.

4 Ukaji, Y.; Ima, M.; Yamada, T.; Inomata, K. Heterocycles 2000, 52, 563.

5 Comins, D. L.; LaMunyon, D. H. J. Org. Chem. 1992, 57, 5807.

6 a) Davis, F. A.; Rao, A.; Carroll, P. J. Org. Lett. 2003, 5, 3855. b) Ranti, H.; Kündig, E. P. Org. Lett. 1999, 1, 1997. c) Comins, D. L.; LaMunyon, D. H. J. Org. Chem. 1992, 57, 5807. 